\title{
miRNA-92a Inhibits the Phenotypic Modulation of Vascular Smooth Muscle Cells and May Help to Prevent In-Stent Restenosis
}

\section{Fenfen Jiang}

The second Affiliated Hospital of Jiaxing University

\section{Bin Zhang}

The second Affiliated Hospital of Jiaxing University

Lang Deng ( $\sim$ langzi213317@126.com )

Affiliated Cent Hospital of HuZhou University

\section{Xiangyu Zhang}

The second Affiliated Hospital of Jiaxing University

\section{Ran Zhang}

The second Affiliated Hospital of Jiaxing University

\section{Qin Lu}

The second Affiliated Hospital of Jiaxing University

\section{Fengjie Shi}

The second Affiliated Hospital of Jiaxing University

Jianjiang $X u$

The second Affiliated Hospital of Jiaxing University

\section{Research Article}

Keywords: in-stent restenosis, vascular smooth muscle cells, coronary atherosclerotic heart disease, miRNA-92a, KLF4

Posted Date: March 7th, 2022

DOI: https://doi.org/10.21203/rs.3.rs-1085399/v1

License: (c) (1) This work is licensed under a Creative Commons Attribution 4.0 International License. Read Full License 


\section{Abstract}

The modulation of the phenotype of vascular smooth muscle cells (VSMCs) during cell proliferation and migration may represent a potential therapeutic approach for the prevention of vascular intimal hyperplasia. However, the precise role of this process in the biology and remodeling of VSMCs remains unclear. Herein, we demonstrate that miR-92a modulates VSMCs to a synthetic phenotype via the KLF4 pathway. Targeting miR-92a in VSMCs with a KLF4 inhibitor suppressed the synthetic phenotype and inhibited the proliferation and migration of VSMCs. To provide definitive evidence, we measured the expression levels of miR-92a in patients undergoing coronary artery intervention; serum miR-92a levels were significantly higher in patients with In-stent restenosis (ISR) than in those without ISR; these findings were the opposite of those obtained for KLF4. Bioinformatics analysis and promoter-luciferase reporter assays were used to examine the regulatory mechanisms underlying KLF4 expression; we found that KLF4 was transcriptionally upregulated by miR-92a in VSMCs. miRNA transfection was also performed to regulate the expression of miR-92a e. We found that the overexpression of miR-92a inhibited the proliferation and migration of VSMCs and also increased differentiated gene and protein expression. Finally, we found that the inhibition of miR-92a promoted the proliferation and migration of VSMCs; this could be reversed by applying a KLF4 inhibitor. Collectively, these results indicate that the local delivery of a KLF4 inhibitor may act as a novel therapeutic option to prevent in-stent restenosis.

\section{Introduction}

Coronary atherosclerotic heart disease (CAHD) is the leading cause of death in the global population (1). With the obesity pandemic and the expected worsening of cardiovascular risk factors in the general population, the incidence and prevalence of heart disease is expected to rise (2). With recent advancements in revascularization techniques and technologies, percutaneous coronary intervention (PCl) and stenting have become the most commonly performed procedures for the treatment of CAHD (3). First generation drug-eluting stents (DES) used were sirolimus (SES; Cypher ${ }^{\mathrm{TM}}$, Cordis Corp, Miami Lakes, FL, USA) and paclitaxel (PES; Taxus ${ }^{\mathrm{TM}}$, Boston Scientific, Natick, Massachusetts), since then, DES have led to a radical reduction in restenosis (4). In-stent restenosis (ISR) can create significant burden on patients undergoing coronary intervention (5). The introduction of DES has significantly lowered target lesion revascularization (TLR) when compared with bare metal stents (BMS); however, $7-10 \%$ of patients need additional procedures within 5 years of treatment and an estimated one in five patients require TLR within 10 years $(6,7)$.

ISR is characterized by the aggregation of platelets, the release of growth factors, inflammatory cell infiltration, VSMCs proliferation and migration, plus the remodeling of extracellular (8). The vascular response to injury depends not only on the cells within the vessels but is also modulated by circulating cells derived from the bone marrow (9). Understanding the molecular mechanisms underlying the physiological healing response and the response to pathological restenosis has become the focus for extensive research. 
Vascular smooth muscle cells (VSMCs) maintain remarkable levels of plasticity during postnatal development and can undergo de-differentiation to a synthetic phenotype (10). This process represents an advantage for survival because it enables the efficient repair of the vasculature following injury (11). As in many evolutionarily conserved processes, these properties can be disadvantageous and can predispose patients to abnormal responses after injury, thus contributing to restenosis $s(8)$. A previous study demonstrated that the recruitment of smooth muscle cells to the intima of the vessel wall was a significant contributor to atherosclerotic plaque progression (12). Activated VSMCs within the medial layer of the vessel wall migrate and proliferate in response to ISR (12). The Krüppel-like factor (KLF) family is paramount in the maintenance of homeostasis in the body, including immune, digestive, respiratory, hematopoietic, and cardiovascular systems (13). KLF members exhibit different biological functions and distinct phenotypes in different diseases, largely resulting from their $\mathrm{N}$-terminal sequences, which provide unique protein interaction motifs and post-translational modification sites (14). KLF4 is expressed in the vascular wall, which includes endothelial cells (ECs) and VSMCs, and plays a critical role in the biology of the vascular wall. KLF4 confers an anti-inflammatory and vasoprotective phenotype to ECs by inhibiting NF-KB activation (15). Previous research has suggested that KLF4 inhibits angiogenesis and endothelial proliferation by mediating microRNAs (miRNAs) in VSMCs $(14,16)$. miRNAs are a class of novel endogenous regulators of gene expression that act at the post-transcriptional level. miRNAs play essential roles in the regulation of many cellular events, such as cell development, differentiation, and apoptosis (17).

miR-92a, a member of the miR17-92 cluster, has been reported to be highly expressed in endothelial cells (18). It has also been reported that miR-92a may play a functional role in VSMCs by protecting against apoptosis induced by oxidative stress (19). However, little is known about the role of miR-92a and the mechanism of its action in the phenotypic modulation of VSMCs. Here, we demonstrate that miR-92a targets KLF4 and plays an important role in promoting the proliferation of VSMCs. We found that miR92a transcription is regulated by KLF4, and that miR-92a and KLF4 form a feedback loop to regulate their own expression and that of VSMCs.

\section{Methods}

\section{Aortic VSMC culture}

Human aortic VSMCs were purchased from ATCC were cultured in DMEM with 10\% FBS and 1\% penicillin/streptomycin in humidified air containing $5 \% \mathrm{CO}_{2}$ under a condition at $37^{\circ} \mathrm{C}(20)$. Cells grown to $80-90 \%$ confluence was passaged at a ratio of $1: 3$. The cells used in all experiments were passaged three to five times.

\section{Patient Sample Collection}

The Second Affiliated Hospital of Jiaxing University (Zhejiang, China) provided the blood samples from CAHD patients after PCl. Details of patients are reported in table 1. blood samples were stored frozen 
until RNA extraction. The informed consents were attained from each patient. All experimental protocols were approved by The Second Affiliated Hospital of Jiaxing University (IRB protocol number: JXEY2021JX083).

Table 1 Baseline characteristics of the study subjects

\begin{tabular}{|llll|}
\hline Variables & Non-ISR $(\mathrm{n}=6)$ & $\mathrm{ISR}(\mathrm{n}=5)$ & $\mathrm{p}$-value \\
\hline Age & $61.33 \pm 9.07$ & $70.2 \pm 8.56$ & 0.168 \\
Gender(male) & 5 & 4 & - \\
\hline BMl $(\mathrm{kg} / \mathrm{m} 2)$ & $23.82 \pm 1.87$ & $24.53 \pm 3.57$ & 0.712 \\
\hline Smoking history & 3 & 3 & - \\
\hline Drinking history & 2 & 2 & - \\
\hline Diabetes & 4 & 1 & - \\
\hline Hypertension & 1 & 4 & - \\
\hline Hyperlipidemia & 0 & 1 & - \\
\hline TC $(\mathrm{mg} / \mathrm{dl})$ & $58.95 \pm 11.40$ & $76.61 \pm 28.02$ & 0.234 \\
\hline TG $(\mathrm{mg} / \mathrm{dl})$ & $29.16 \pm 9.24$ & $30.64 \pm 11.11$ & 0.833 \\
\hline HDL-C $(\mathrm{mg} / \mathrm{dl})$ & $17.01 \pm 3.31$ & $16.45 \pm 3.50$ & 0.812 \\
\hline LDL-C $(\mathrm{mg} / \mathrm{dl})$ & $28.65 \pm 8.56$ & $41.22 \pm 19.01$ & 0.221 \\
\hline
\end{tabular}

\section{Western Blotting}

RIPA buffer containing protease inhibitors (Beyotime, China) was used to extract Total protein. $30 \mu \mathrm{g}$ per lane protein was separated on a 10\% SDS-PAGE gel, and transferred onto a $0.45 \mu \mathrm{m}$ PVDF membrane (Millipore, USA). After blocking with $5 \%$ non-fat dry milk, the membrane was separately incubated in $4{ }^{\circ} \mathrm{C}$ overnight with Mhc Polyclonal Antibody (1:500 dilution, Proteintech, 10799-1-AP, USA), Smooth Muscle Actin Polyclonal Antibody (1:1500 dilution, Proteintech, 14395-1-AP, USA), Osteopontin Polyclonal Antibody (1:500 dilution, Proteintech, 25715-1-AP, USA) or GAPDH Polyclonal Antibody (1:8000 dilution, Proteintech, G9545, USA) antibodies. Next day, the PVDF membrane was washed three time and soaked in secondary antibody (Jackson, USA) for two hours at room temperature. The proteins were detected with the ECL reagents.

\section{Real-Time PCR Assay}


RT-PCR was performed using the 2x SYBR Green Master Mix (US EVERBRIGHT, China) for 45 cycles in a total volume of $20 \mu \mathrm{l}$. All reactions were performed in duplicate. While mRNA expression levels were normalized to GAPDH using the $2^{-\triangle \triangle C T}$ method. In all sequences for each primer in regards to a-SMA, SM$\mathrm{MHC}, \mathrm{OPN}$ and GAPDH were as follows:

a-SMA, TGTTCCAGCCGTCCTTCATC and GGGAGCCAAAGCAGTGATCT; SM-MHC, CGAAGGGCTTGAATGAGGAGT and GCTTCCTCCCAAGGAGCTGTAT; OPN, GAGGAAAAGGAGACCCTTCCA and TGAAAACTTCGGTTGCTGGC; GAPDH, ACAGTCAGCCGCATCTTCTT and GACTCCGACCTTCACCTTCC.

\section{Dual-Luciferase Reporter Assay}

Relative luciferase activity was detected using a Dual-Luciferase Assay Kit (Promega, USA). Bioinformatics analysis performed by TargetScan revealed that KLF4 containted the binding site of miR92a at the 3'-UTR. The mutant 3' UTR of KLF4 was amplified using the pGL3/Luc- KLF4 3' UTR as the template and was cloned downstream of the pGL3/Luc vector. VSMCs were planted in 96-well plates and co-transfected with either $100 \mathrm{nM}$ of miR-92a mimic or control and 2 $\mu \mathrm{g}$ pGL3/Luc- KLF4 3' UTR or the mutant 3' UTR using Lipofectamine ${ }^{\circledR} 2000$ reagent according to the manufacturer's protocol.

$48 \mathrm{~h}$ later, Luciferase intensity were assessed using the Dual Luciferase Reporter 1000 Assay System (Promega, USA).

\section{MTT assay}

VSMCs transfected with a miR-92a mimic, inhibitor or control were planted in 96-well plates with adjusted density of 7,000 cells per well, at $37^{\circ} \mathrm{C}$ under $5 \%$ CO2 for $24,48,72$ and 96 h. 3-(4,5-dimethylthiazol-2yl)-2,5-diphenyl tetrazolium bromide (MTT, Sigma-Aldrich, USA) diluted in DMEM was added into the medium with a final concentration of $5 \mathrm{mg} / \mathrm{mL}$ to each well for a further $4 \mathrm{~h}$. The supernatant was carefully removed, and dimethyl sulfoxide $(200 \mu \mathrm{L})$ was added to each well. The suspension was placed in a dark place for $2 \mathrm{~h}$ at room temperature and the absorbance was measured at $570 \mathrm{~nm}$ with an absorbance reader (Thermo Fisher Scientific, USA).

\section{Transwell Invasion Assays}

Cell invasion were assessed using Transwell chambers with a pore size of $8 \mu \mathrm{m}$ (Corning, USA). Briefly, after transfection with miR-92a mimic or miR-92a inhibitor, $1 \times 10^{5}$ VSMCs were added to the upper chamber in serum-free DMEM medium. After 0, 24, 48, 72 and $96 \mathrm{~h}$, the insert membranes were fixed in chilled methanol and incubated in $0.5 \%$ crystal violet. The number of invading cells was counted under an inverted microscope and photographed.

\section{Statistical Analysis}


All results were shown as the mean \pm standard deviation. The statistical analysis was analyzed using SPSS version 13.0 and GraphPad Prism software. ImageJ software was used to deal with image analysis. Statistical significance of the differences between groups were calculated using ANOVA or the two-tailed Student's t-test. $p<0.05$ was considered as statistically significant differences. All experiments were repeated three times.

\section{Results}

\section{The association between miR-92a and KLF4 in patients with ISR}

Analysis showed that the levels of miR-92a were lower in patients with ISR patients $(n=5)$ than in control individuals $(n=5)$ (Figure 1A) and were inversely correlated with the expression of KLF4 in patients with ISR (Figure 1B), as determined by Pearson's correlation model (Figure 1C).

\section{Figure 1}

\section{miR-92a promoted the expression of KLF4 by targeting its 3'-UTR}

Studies have shown that KLF4 is an antiproliferative regulator for VSMCs. To test whether KLF4 is a direct target of miR-92a, we used a bioinformatics approach to search for the potential matching site of miR-92a in the KLF4 3'-UTR. We successfully identified a binding site for miR-92a at nucleotides 674 681 of the homo sapiens KLF4 3'-UTR (Figure 2), thus suggesting that this miRNA plays an important role in the regulation of gene expression.

\section{Figure 2}

\section{miR-92a contributed to the synthetic phenotype switching of cerebral VSMCs}

To determine whether miR-92a modulates the phenotypes of VSMCs, we detected the protein levels of contractile and synthetic markers for cerebral VSMCs.

For

In mature and normal blood vessels, VSMCs has been shown to be a highly quiescent and contractile phenotype associated with elevated levels of contractile markers proteins such as a-smooth muscle actin (a-SMA) and smooth muscle myosin heavy chain (SMMHC) (21). In atherosclerosis and arterial restenosis, VSMCs can switch to a de-differentiated, proliferative, and migratory phenotype by downregulating the gene expression of VSMC contractile markers and by upregulating the synthetic protein expression of osteopontin (OPN) $(22,23)$. Compared with controls, the expression levels of a-SMA and SMMHC were lower in the miR-92a inhibitor group (Figure 3A-C, E-G) while the OPN levels were higher in the miR-92a inhibitor group (Figure 3D and $\mathrm{H}$ ). Collectively, these results demonstrated that miR-92a could promote the phenotypic modulation of VSMCs.

\section{Figure 3}




\section{miR-92a promoted the phenotypic modulation of VSMCs}

To evaluate the functional effects of miR-92a, we transfected cells with a. miR-92a mimic for 24 hours and observed an increase in VSMC proliferation (Figure 4A) and migration (Figure 4B).

\section{Figure 4}

\section{HY-12302 promoted synthetic phenotype switching in cerebral VSMCs}

We postulated that KLF4 might partially mediate the effects of miR-92a on VSMCs. Kenpaullone (HY12302), a small molecule inhibitor of KLF4, along with miR-92a inhibitors, were used in functional assays involving VSMCs. Following transfection with a miR-92a inhibitor, VSMCs showed increased expression of the synthetic gene OPN along with decreased expression of the contractile genes (a-SMA and SMMHC) at both the mRNA (Figure 5A-C) and protein levels (Figure 5D-G). These results showed that the miR-92a inhibitor promoted the phenotypic alteration in VSMCs from differentiated to dedifferentiated cells, and that treatment with the KLF4 inhibitor HY-12302 antagonized the dedifferentiation response of VSMCs to the miR-92a inhibitor (Figure 5).

\section{Figure 5}

\section{HY-12302 partially abrogated the migration and proliferation of VSMCs}

The MTT cell proliferation assay was used to determine the proliferation rate of VSMCs in each group. The KLF4 inhibitor HY-12302 partially abrogated the accelerated cell proliferation (Figure 6A) and invasion (Figure 6B) induced by the miR-92a inhibitor.

\section{Figure 6}

\section{Discussion}

Our primary novel findings in this study are that treatment with a KLF4 inhibitor (HY-12302) attenuated miR-92a inhibitor-induced VSMC differentiation, proliferation, and migration. These results provide evidence for the molecular mechanisms underlying the protective role of miR-92a in VSMCs.

Revascularization remains the cornerstone for managing patients with CAHD. Studies identified PCI and the insertion of metal scaffolds has emerged as one of the most preferred method for restoring vessel patency, while $30 \%$ of patients will experience a gradual re-narrowing of the lumen caused by neointima (NI) formation, which is resulting ISR (24).

It has been reported that the causes of ISR are the appearance of VSMCs and myofibroblasts which produce an abundant extra-cellular matrix $(\mathrm{ECM})(25,26)$.

VSMCs can be induced by various environmental stimuli, including vascular injury, growth factors, and inflammatory stimuli. Those condition switch VSMCs from a quiescent "contractile" phenotype to an 
active "synthetic" mode (27). Mature VSMCs show a contractile phenotype, characterized by a low proliferation and a high protein expression of SMMHC, a-SMA, and calponin; these proteins are required for contractile functionality. While during vascular injury, such as angioplasty or bypass surgery, VSMCs can dedifferentiate to a synthetic phenotype, characterized by a high proliferation, migration, the production of ECM components, and the reduction of VSMC-specific markers (28). Synthetic VSMCs migrate and proliferate, and their accumulation over time can lead to neointima formation $(29,30)$. As such, the inhibition of inflammation in VSMCs might be an important step to limit ISR in a clinical scenario. The current study found that increased levels of VSMC synthetic protein expression and proliferation in the presence of miR-92a mimics, demonstrating that miR-92a is a positive regulator for the VSMC synthetic phenotype in vitro.

KLF4 plays an important role in several vascular diseases and is expressed by different cell types involved in the development of vascular disease, including VSMCs (31). A previous study identified KLF4 as a transcriptional target to modulate the differentiation of VSMCs. KLF4 is not normally expressed in differentiated VSMCs in vivo, but is transiently induced in VSMCs after vascular injury (32). Of interest, we found that the expression levels of KLF4 were increased in ISR patients while the expression levels of miR-92a were decreased. Furthermore, miR-92a levels were negatively correlated with KLF4. Collectively, our data indicated that KLF4 is a direct target of miR-92a. This indicates that specific mediation of the VSMC-miR-92a-KLF4 pathway might represent an effective therapy option to mitigate ISR in the clinic. miRNAs are a class of non-coding small RNA molecules that play important roles in regulating posttranscriptional gene expression. In most cases, miRNAs interact with the 3'-UTR of target mRNAs to inhibit gene translation or induce mRNA degradation (33). miR-92a has been reported to be an oncogene in many different forms of tumors (34). It has also been reported that miR-92a is a negative regulator of endothelial function and angiogenesis (35). However, our current data showed that miR-92a is a positive regulator for the synthetic phenotype in VSMCs and acts by modulating KLF4.

In conclusion, we elucidated a VSMC-miR-92a-KLF4 pathway for mediating the negative regulation of VSMC differentiation in ISR. As vascular injury induces KLF4 expression and activation, the overexpression of miR-92a, or treatment involving the KLF4 inhibitor (HY-12302), can protect against injuryinduced VSMC polarization. These effects may be attributed to the increased contractile protein expression in VSMCs and the inhibition of proliferation and migration. Given that levels of miR-92a are significantly reduced in ISR patients, our present findings laid down an important mechanistic foundation for therapeutically targeting ISR with a KLF4 inhibitor.

\section{Declarations}

\section{Ethics approval and consent to participate}

The Second Affiliated Hospital of Jiaxing University (Zhejiang, China) provided the blood samples from $\mathrm{CAHD}$ patients after $\mathrm{PCl}$. The informed consents were attained from each patient. All experimental protocols were approved by The Second Affiliated Hospital of Jiaxing University (IRB protocol number: 
JXEY-2021JX083), and all methods were carried out in accordance with relevant guidelines and regulations. This article does not contain any studies with animals.

\section{Consent for publication}

Not applicable

\section{Competing interests}

The authors declare that they have no competing interests

\section{Funding}

This study was supported by the Science and Technology Bureau of Jiaxing City (2021AD30103, 2020AD30119, 2021AD30090)

\section{Authors' contributions}

FJ and BZ designed the experiments, interpreted the results and wrote the manuscript; DL supervised the project, analyzed the data and wrote the paper; The rest of the authors helped to design and generate the experiments.

\section{Acknowledgements}

Not applicable

\section{Data availability statement}

The datasets generated and analysed during the current study are available in the figshare repository, [https://figshare.com/s/b7523e4de4cd36393f11]

\section{References}

1. Malakar AK, Choudhury D, Halder B, Paul P, Uddin A, Chakraborty S. A review on coronary artery disease, its risk factors, and therapeutics. J Cell Physiol. 2019;234(10):16812-23.

2. Faroux L, Guimaraes L, Wintzer-Wehekind J, Junquera L, Ferreira-Neto AN, Del Val D, et al. Coronary Artery Disease and Transcatheter Aortic Valve Replacement: JACC State-of-the-Art Review. J Am Coll Cardiol. 2019;74(3):362-72.

3. Al-Lamee RK, Nowbar AN, Francis DP. Percutaneous coronary intervention for stable coronary artery disease. Heart. 2019;105(1):11-9.

4. Davis AA, Patel VG. The role of PD-L1 expression as a predictive biomarker: an analysis of all US Food and Drug Administration (FDA) approvals of immune checkpoint inhibitors. J Immunother Cancer. 2019;7(1):278. 
5. Ali RM, Abdul Kader MASK, Wan Ahmad WA, Ong TK, Liew HB, Omar AF, et al. Treatment of Coronary Drug-Eluting Stent Restenosis by a Sirolimus- or Paclitaxel-Coated Balloon. JACC Cardiovasc Interv. 2019;12(6):558-66.

6. Alfonso F, Byrne RA, Rivero F, Kastrati A. Current treatment of in-stent restenosis. J Am Coll Cardiol. 2014;63(24):2659-73.

7. Lansky A, Grubman D, Scheller B. Paclitaxel-coated balloons: a safe alternative to drug-eluting stents for coronary in-stent restenosis. Eur Heart J. 2020;41(38):3729-31.

8. Marx SO, Totary-Jain H, Marks AR. Vascular smooth muscle cell proliferation in restenosis. Circ Cardiovasc Interv. 2011;4(1):104-11.

9. Majesky MW. Vascular Development. Arterioscler Thromb Vasc Biol. 2018;38(3):e17-e24.

10. Touyz RM, Alves-Lopes R, Rios FJ, Camargo LL, Anagnostopoulou A, Arner A, et al. Vascular smooth muscle contraction in hypertension. Cardiovasc Res. 2018;114(4):529-39.

11. Wang Y, Nanda V, Direnzo D, Ye J, Xiao S, Kojima Y, et al. Clonally expanding smooth muscle cells promote atherosclerosis by escaping efferocytosis and activating the complement cascade. Proc Natl Acad Sci U S A. 2020;117(27):15818-26.

12. Caplice NM, Bunch TJ, Stalboerger PG, Wang S, Simper D, Miller DV, et al. Smooth muscle cells in human coronary atherosclerosis can originate from cells administered at marrow transplantation. Proc Natl Acad Sci U S A. 2003;100(8):4754-9.

13. Fan Y, Lu H, Liang W, Hu W, Zhang J, Chen YE. Krüppel-like factors and vascular wall homeostasis. J Mol Cell Biol. 2017;9(5):352-63.

14. McConnell BB, Yang VW. Mammalian Krüppel-like factors in health and diseases. Physiol Rev. 2010;90(4):1337-81.

15. Hamik A, Lin Z, Kumar A, Balcells M, Sinha S, Katz J, et al. Kruppel-like factor 4 regulates endothelial inflammation. J Biol Chem. 2007;282(18):13769-79.

16. Zheng X, Li A, Zhao L, Zhou T, Shen Q, Cui Q, et al. Key role of microRNA-15a in the KLF4 suppressions of proliferation and angiogenesis in endothelial and vascular smooth muscle cells. Biochem Biophys Res Commun. 2013;437(4):625-31.

17. Sun SG, Zheng B, Han M, Fang XM, Li HX, Miao SB, et al. miR-146a and Krüppel-like factor 4 form a feedback loop to participate in vascular smooth muscle cell proliferation. EMBO Rep. 2011;12(1):5662.

18. Sand M, Hessam S, Amur S, Skrygan M, Bromba M, Stockfleth E, et al. Expression of oncogenic miR17-92 and tumor suppressive miR-143-145 clusters in basal cell carcinoma and cutaneous squamous cell carcinoma. J Dermatol Sci. 2017;86(2):142-8.

19. Zhang L, Zhou M, Wang Y, Huang W, Qin G, Weintraub NL, et al. miR-92a inhibits vascular smooth muscle cell apoptosis: role of the MKK4-JNK pathway. Apoptosis. 2014;19(6):975-83.

20. Jay DB, Papaharalambus CA, Seidel-Rogol B, Dikalova AE, Lassegue B, Griendling KK. Nox5 mediates PDGF-induced proliferation in human aortic smooth muscle cells. Free Radic Biol Med. 
2008;45(3):329-35.

21. Liao XH, Wang N, Zhao DW, Zheng DL, Zheng L, Xing WJ, et al. STAT3 Protein Regulates Vascular Smooth Muscle Cell Phenotypic Switch by Interaction with Myocardin. J Biol Chem. 2015;290(32):19641-52.

22. Bennett MR, Sinha S, Owens GK. Vascular Smooth Muscle Cells in Atherosclerosis. Circ Res. 2016;118(4):692-702.

23. Lu QB, Wan MY, Wang PY, Zhang CX, Xu DY, Liao X, et al. Chicoric acid prevents PDGF-BB-induced VSMC dedifferentiation, proliferation and migration by suppressing ROS/NFKB/mTOR/P70S6K signaling cascade. Redox Biol. 2018;14:656-68.

24. O' Brien ER, Ma X, Simard T, Pourdjabbar A, Hibbert B. Pathogenesis of neointima formation following vascular injury. Cardiovasc Hematol Disord Drug Targets. 2011;11(1):30-9.

25. Glover $\mathrm{C}, \mathrm{Ma} X$, Chen $\mathrm{YX}$, Miller H, Veinot J, Labinaz M, et al. Human in-stent restenosis tissue obtained by means of coronary atherectomy consists of an abundant proteoglycan matrix with a paucity of cell proliferation. Am Heart J. 2002;144(4):702-9.

26. Glover C, O'Brien ER. Pathophysiological insights from studies of retrieved coronary atherectomy tissue. Semin Interv Cardiol. 2000;5(4):167-73.

27. Sorokin V, Vickneson K, Kofidis T, Woo CC, Lin XY, Foo R, et al. Role of Vascular Smooth Muscle Cell Plasticity and Interactions in Vessel Wall Inflammation. Front Immunol. 2020;11:599415.

28. Shi J, Yang Y, Cheng A, Xu G, He F. Metabolism of vascular smooth muscle cells in vascular diseases. Am J Physiol Heart Circ Physiol. 2020;319(3):H613-H31.

29. Wu JH, Zhou YF, Hong CD, Chen AQ, Luo Y, Mao L, et al. Semaphorin-3A protects against neointimal hyperplasia after vascular injury. EBioMedicine. 2019;39:95-108.

30. Wu W, Zhang W, Choi M, Zhao J, Gao P, Xue M, et al. Vascular smooth muscle-MAPK14 is required for neointimal hyperplasia by suppressing VSMC differentiation and inducing proliferation and inflammation. Redox Biol. 2019;22:101137.

31. Liao X, Sharma N, Kapadia F, Zhou G, Lu Y, Hong H, et al. Krüppel-like factor 4 regulates macrophage polarization. J Clin Invest. 2011;121(7):2736-49.

32. Ghaleb AM, Yang VW. Krüppel-like factor 4 (KLF4): What we currently know. Gene. 2017;611:27-37.

33. O'Brien J, Hayder H, Zayed Y, Peng C. Overview of MicroRNA Biogenesis, Mechanisms of Actions, and Circulation. Front Endocrinol (Lausanne). 2018;9:402.

34. Bartel DP. MicroRNAs: genomics, biogenesis, mechanism, and function. Cell. 2004;116(2):281-97.

35. Liu PJ, Ye YX, Wang YX, Du JX, Pan YH, Fang XB. MiRNA-92a promotes cell proliferation and invasion through binding to KLF4 in glioma. Eur Rev Med Pharmacol Sci. 2019;23(15):6612-20.

\section{Figures}



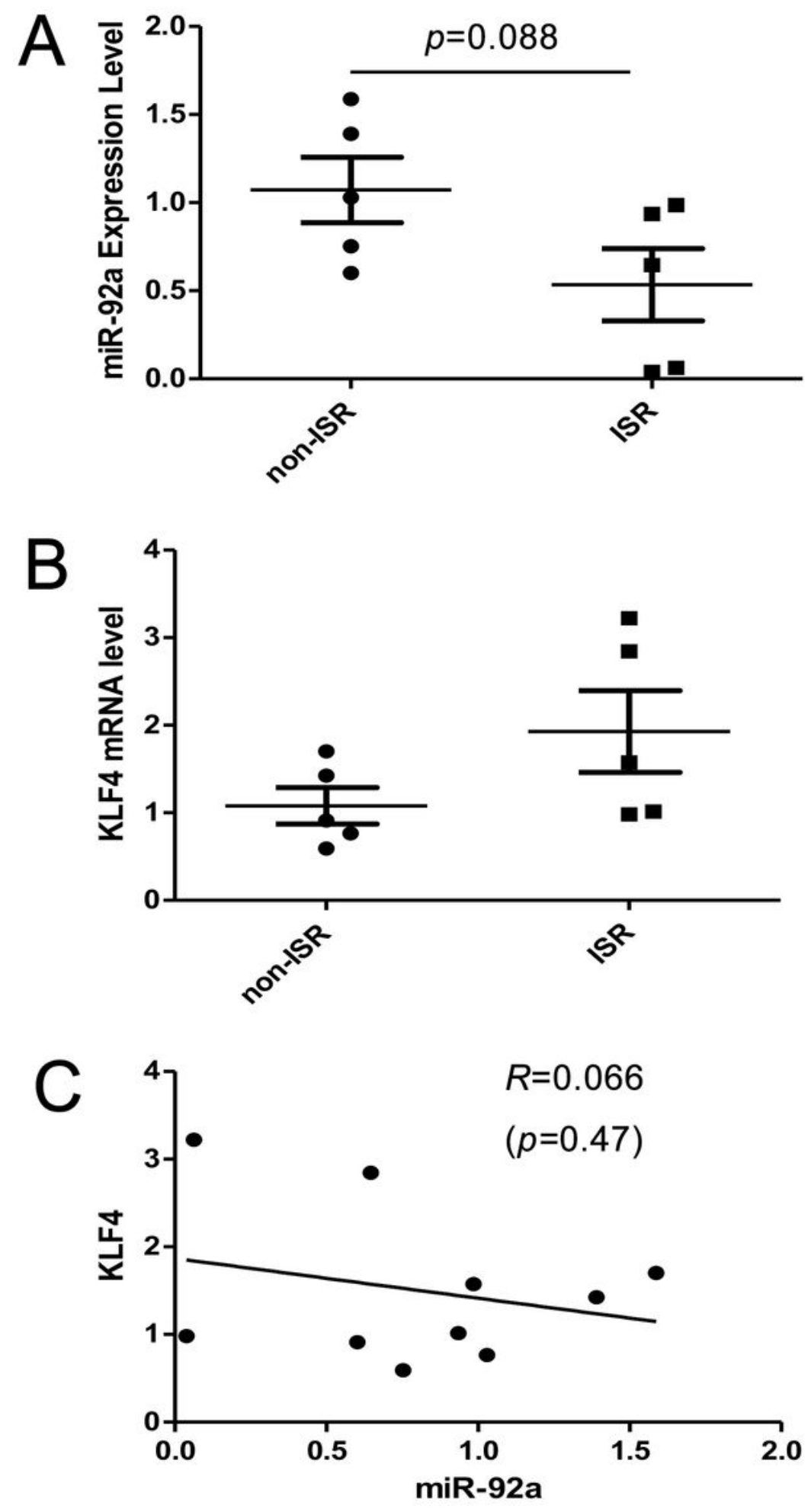

Figure 1

miR-92a and KLF4 mRNA expression levels in ISR patients. (A) miR-92a levels were significantly lower in ISR patients $(n=5)$ than in control subjects without ISR $(n=5)$. Each point represents the expression level of miR-92a from individual patients or control subjects. (B) KLF4 levels were lower in ISR patients $(n=5)$ than in control subjects without ISR $(n=5)$. (C) miR-92a levels were negatively correlated with KLF4 in ISR patients. 


\section{A}

Position 674-681 of KLF4 3' UTR 5'-ACUGUGGUUUCAGAUGUGCAAUA-3' hsa-miR-92a 3'-UGUCCGGCCCUGUUCACGUUAU-5'

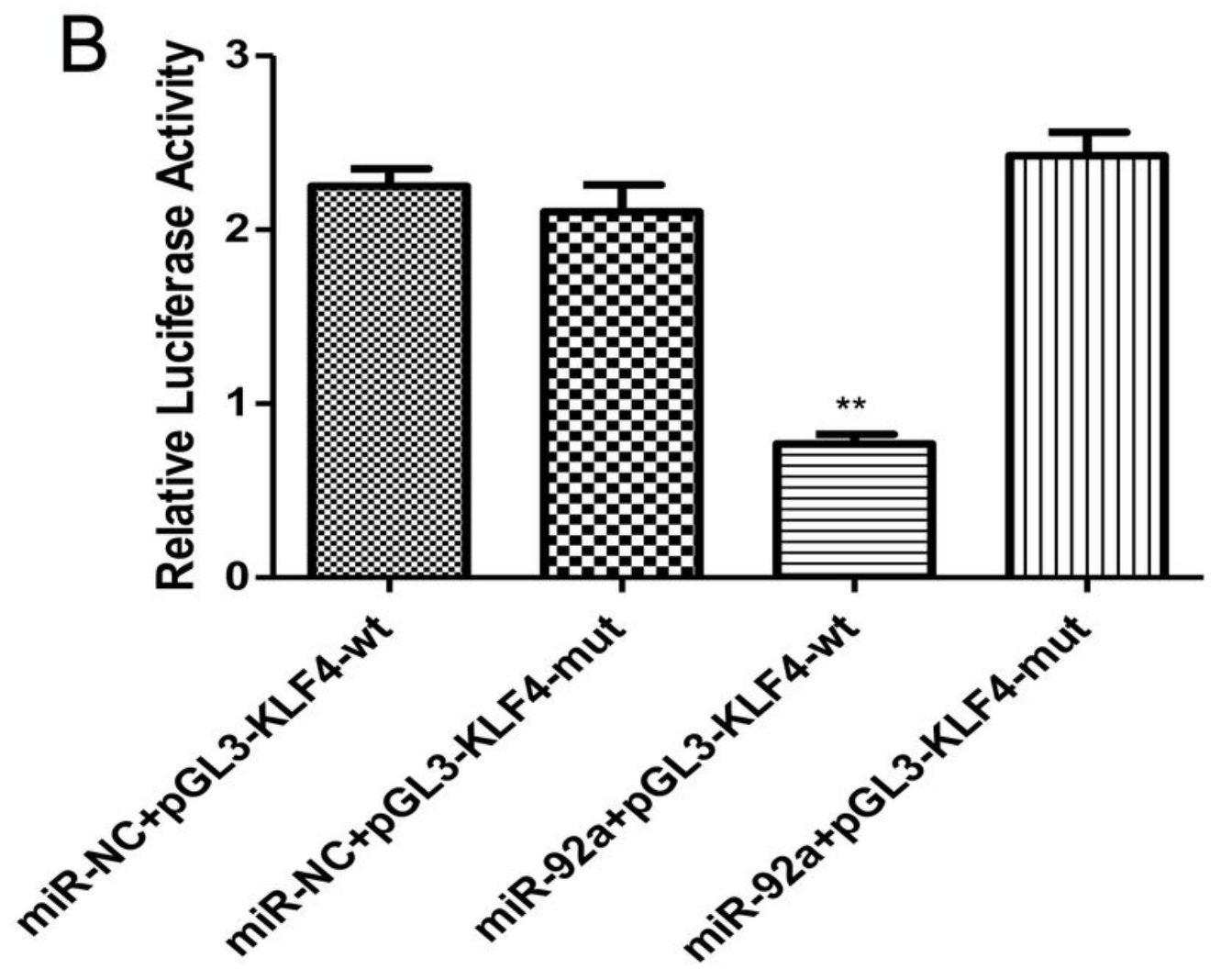

Figure 2

(A) The binding site for miR-92a is located at nucleotides 674 - 681 of the homo sapiens KLF4 mRNA 3'UTR and is highly conserved in vertebrates. (B) VSMCs were co-transfected with pGL3-miR-92a, the wildtype KLF4-UTR-WD, or with its mutant (KLF4-UTR-MT). After $24 \mathrm{~h}$, we measured luciferase activity. * $\mathrm{p}<$ $0.05, \star \star p<0.01 . n=3$ for each group. 

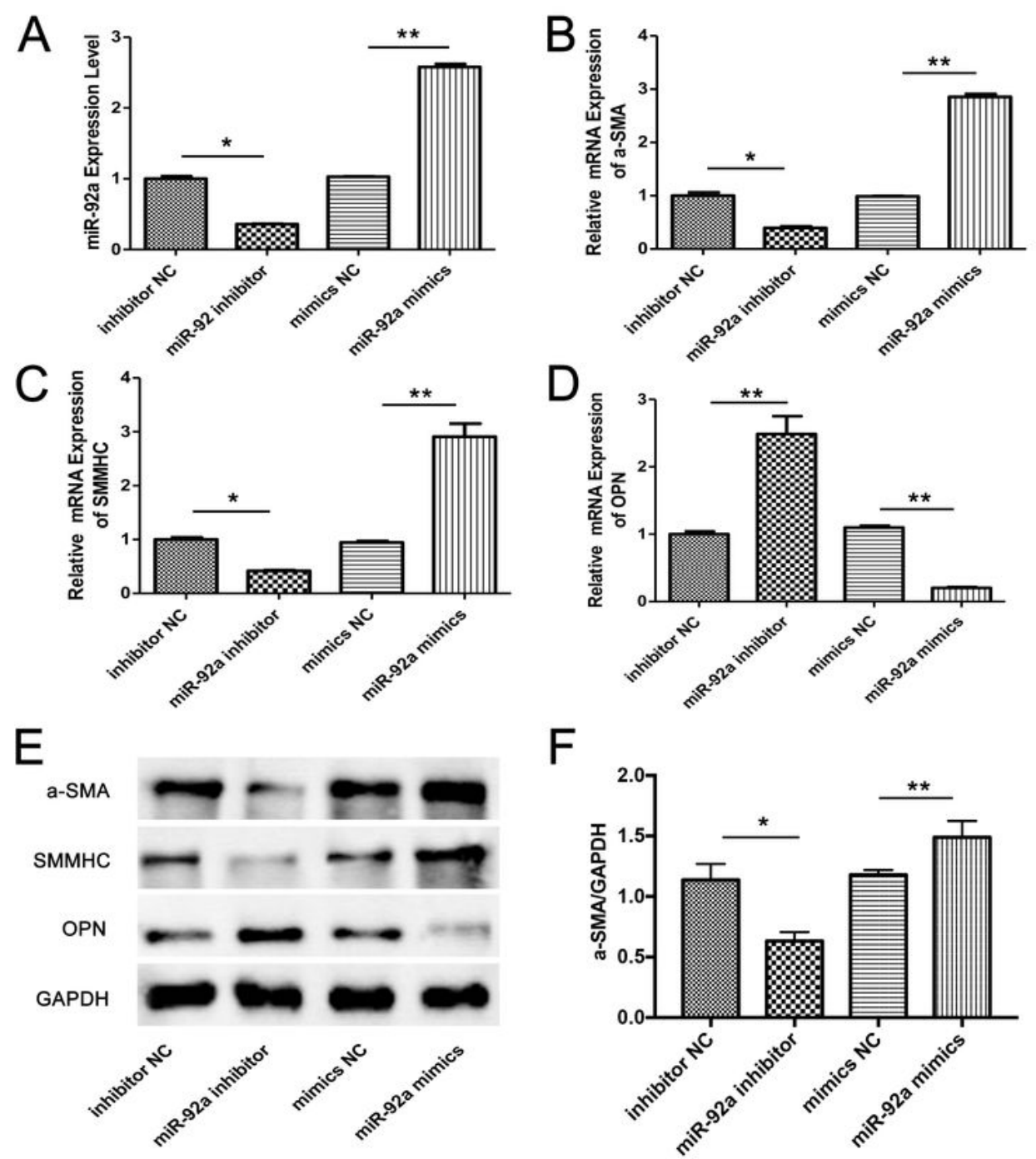

$\mathrm{F}$
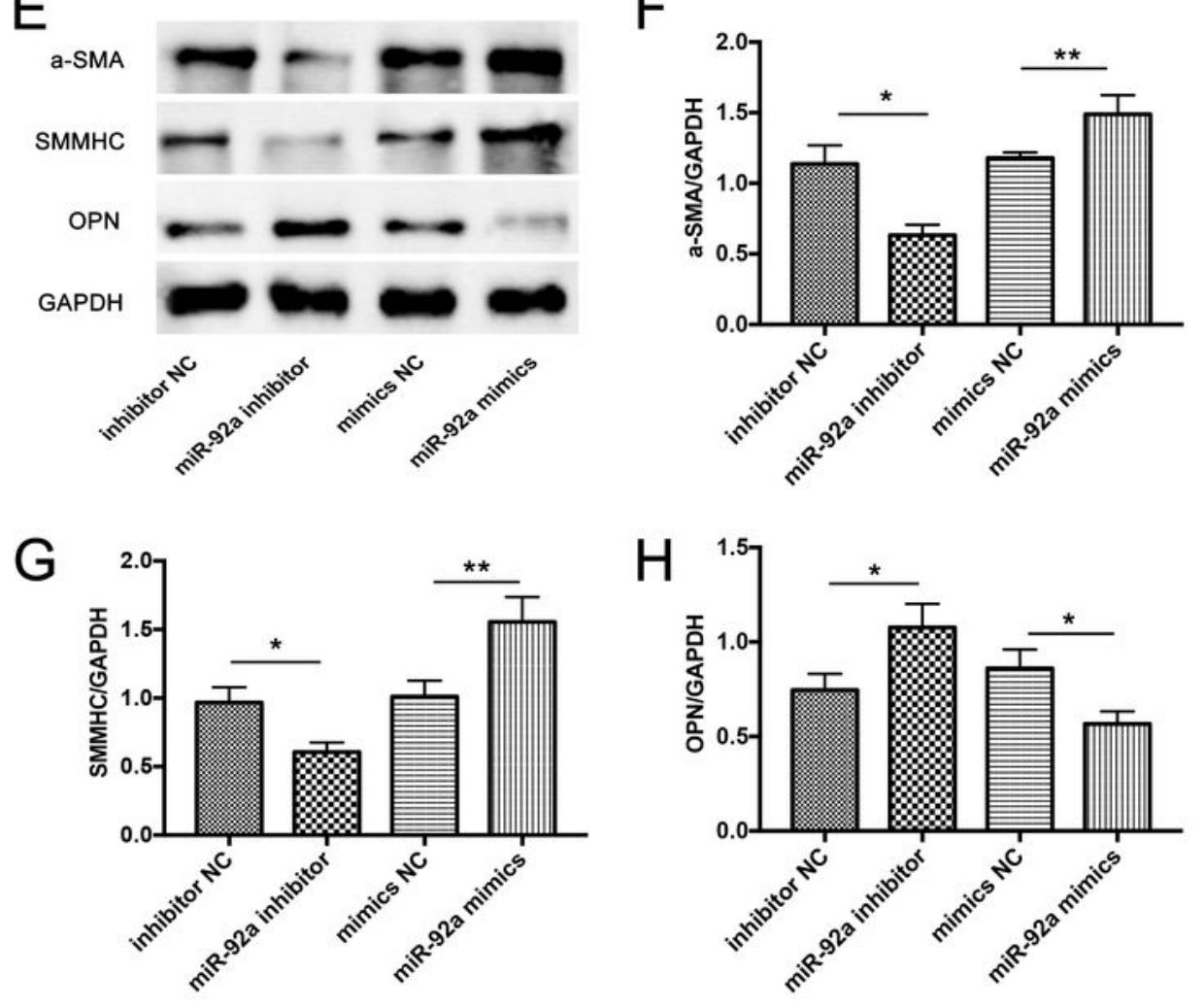

Figure 3

(A) The expression levels of miR-92a in VSMCs transfected with a miR-92a mimic or inhibitor. (B-H) Western blot analysis of contractile and synthetic markers of VSMCs. ${ }^{*} p<0.05,{ }^{*} p<0.01$. $n=3$ for each group. 

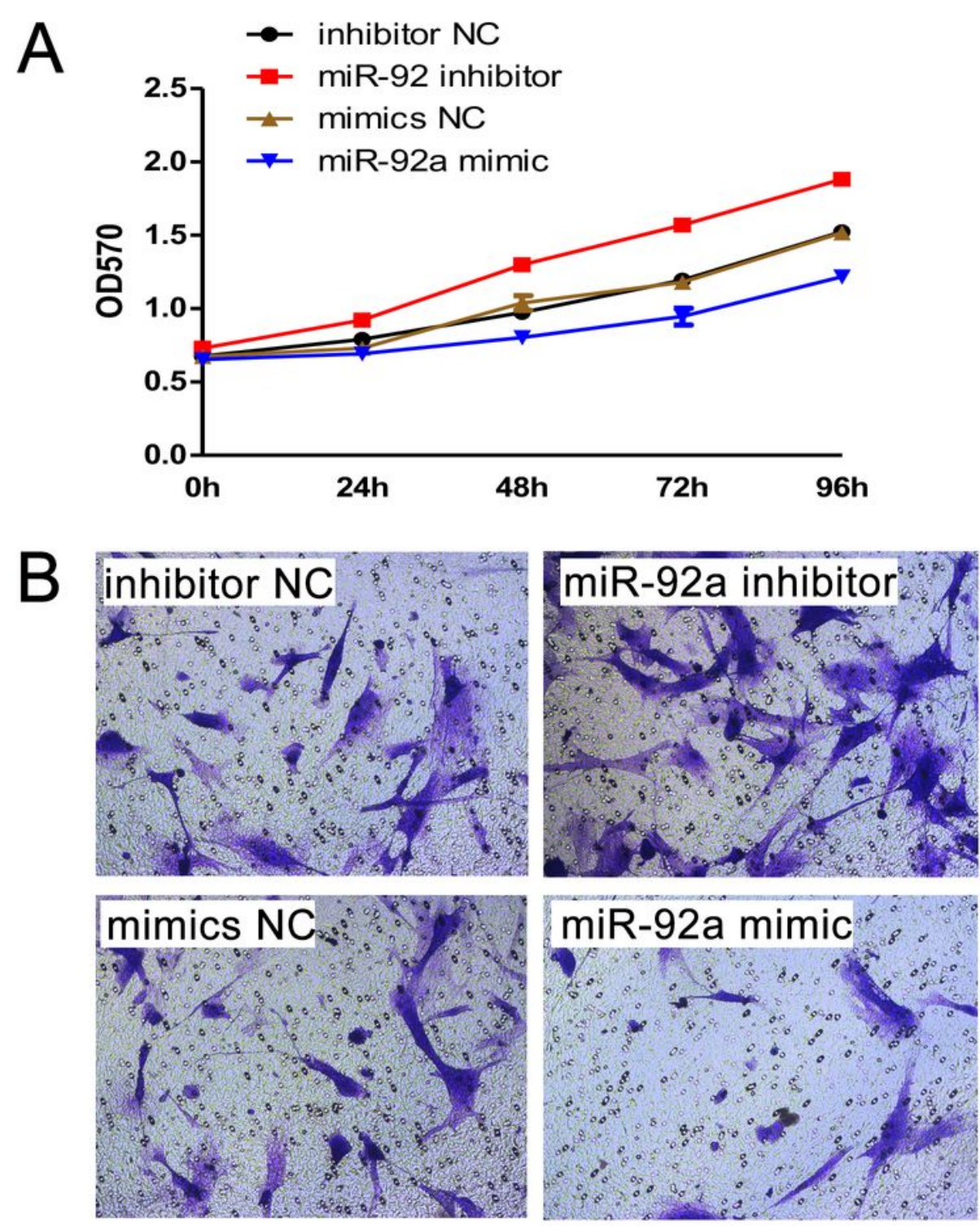

Figure 4

(A) The proliferation of VSMCs was evaluated by MTT assays in VSMCs transfected with a miR-92a mimic or inhibitor. (B) The migration of VSMCs was measured using Transwell plates and VSMCs transfected with a miR-92a mimic or inhibitor. ${ }^{*} p<0.05,{ }^{* \star} p<0.01 . n=3$ for each group. 
A

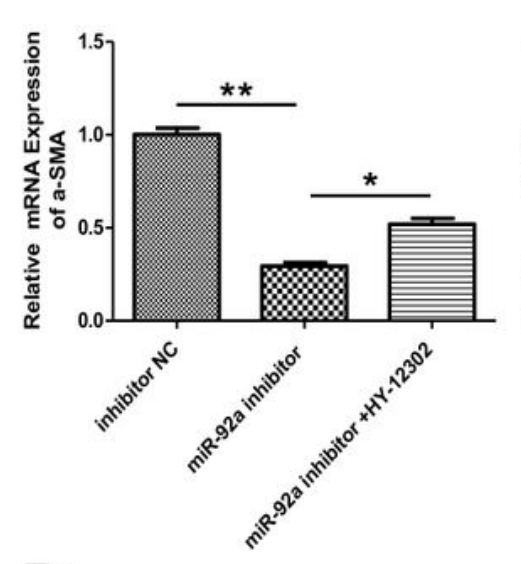

D
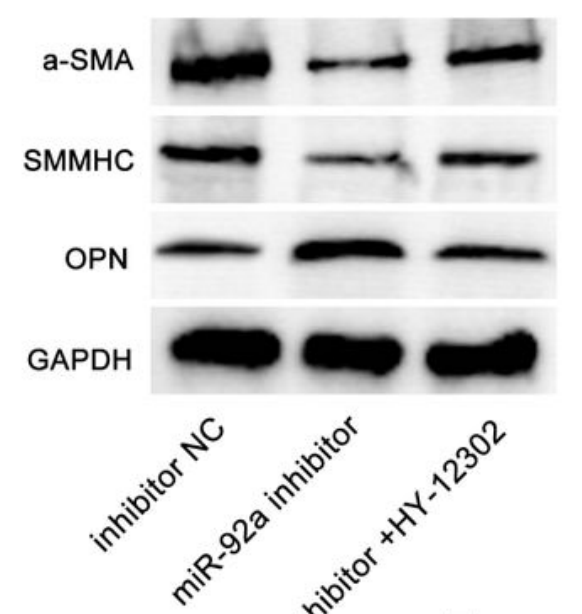
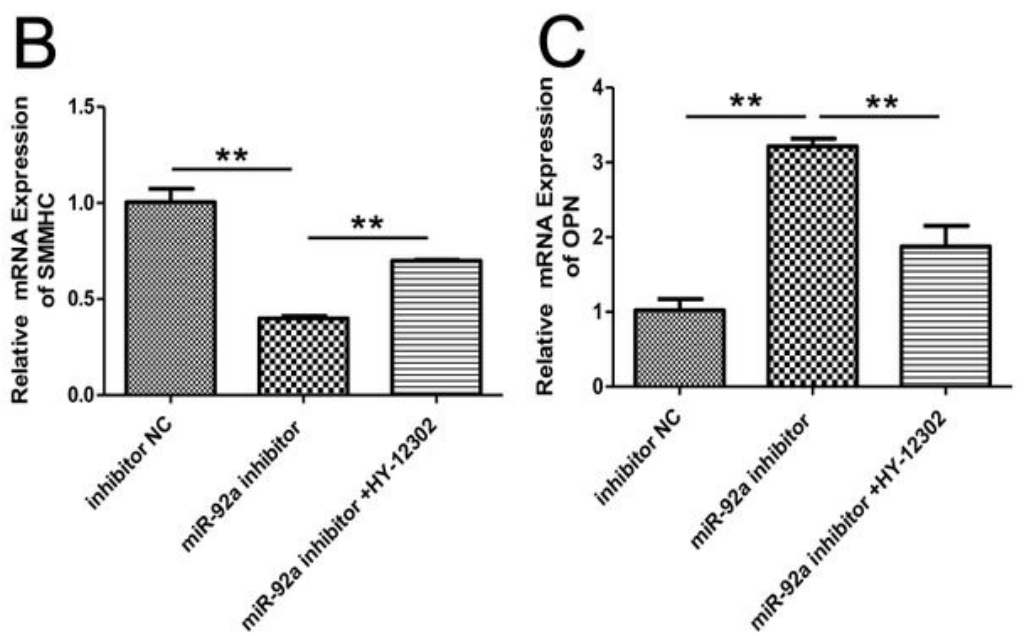

E

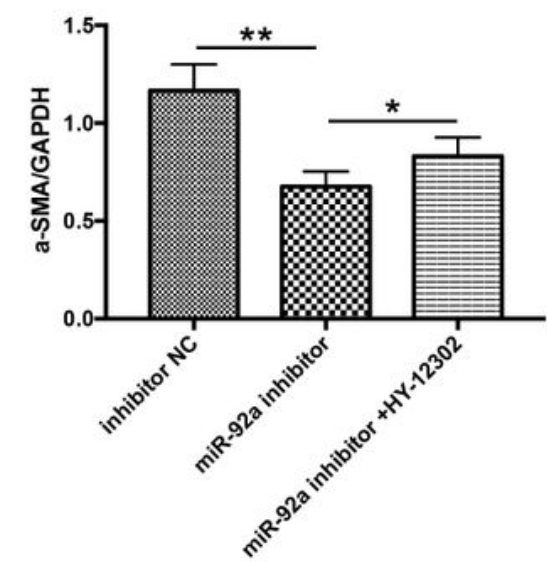

G

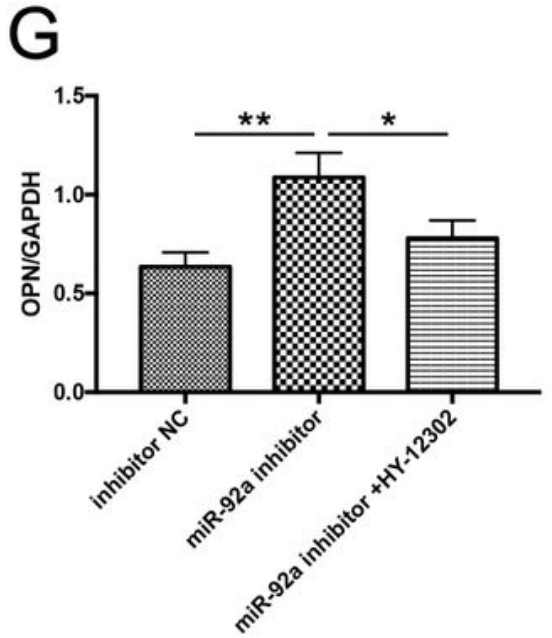

\section{Figure 5}

HY-12302 abrogated miR-92a inhibitor-induced VSMC dedifferentiation. (A-C) Bar graph showing the relative mRNA levels of a-SMA, SMMHC, and OPN. (D) Western blotting was used to was determine the expression levels of contractile proteins (a-SMA and SMMHC) and the OPN synthetic proteins. (E-G) Bar graph showing the relative protein level of a-SMA, SMMHC, and OPN. * $p<0.05, * \star p<0.01$. $n=3$ for each group. 

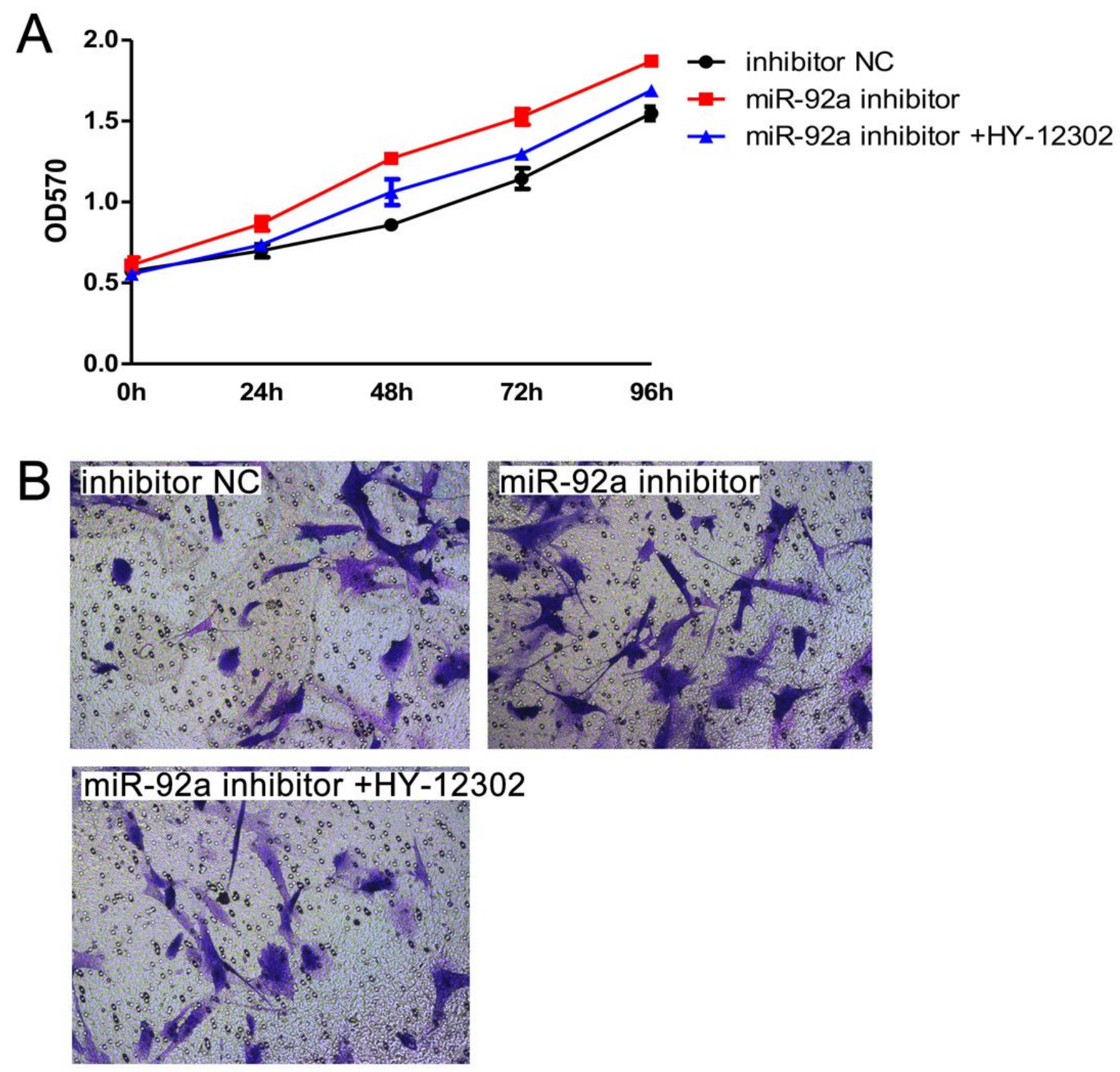

\section{Figure 6}

The proliferation and migration of VSMCs was abrogated by KLF4 inhibition. (A) The suppressive effect on cell proliferation elicited by the miR-92a inhibitor was alleviated by HY-12302. Cell viability was monitored at $0,24,48,72$ and $96 \mathrm{~h}$ by the MTT assay. (B) Representative results from Transwell plates showing the effects of miR-92a inhibitor and HY-12302 on the migration of VSMCs as compared to basal conditions. ${ }^{*} \mathrm{p}<0.05,{ }^{\star *} \mathrm{p}<0.01$. $\mathrm{n}=3$ for each group. 


\section{Supplementary Files}

This is a list of supplementary files associated with this preprint. Click to download.

- supplementaryinformationmiRNA92.pdf 